\title{
Investigation of Chitosan Embedded with Silver Vanadate Nanorods and their Optical Properties
}

\author{
A.M. Abdelghany ${ }^{*}$, M.Y.A. Abdelaal ${ }^{2}$, D.M. Ayaad ${ }^{2}$, A.M. Aboelkheir ${ }^{2}$ \\ ${ }^{1}$ Spectroscopy Department, Physics Division, National Research Center, 33 Elbehouth \\ st., 12311, Dokki, Giza and ${ }^{2}$ Chemistry Department, Faculty of Science, Mansoura \\ University, Mansoura, 35516, Egypt.
}

\begin{abstract}
CHITOSAN is a natural polysaccharide polymer which is non-toxic, biodegradable, cationic and biocompatible. Present work study physical and chemical characteristics of pure chitosan thin film and other samples that modified through embedding different concentration of silver vanadate nanorods prepared via solution casting route. Casting route was employed for preparation of chitosan thin films and other sample containing gradient increase of synthesized silver vanadate nanorods.The optical properties have been investigated through ultraviolet-visible (UV/Vis) optical absorption spectroscopy. Experimental data reveals an observable decrease in the values of optical energy gap with increasing content of silver vanadate nanorods which result in enhancement of the sensitivity for all prepared thin films.
\end{abstract}

Keywords: Nanocomposites; Chitosan; Semiconductors; Silver vanadate; $\mathrm{UV} / \mathrm{Vis}$

\section{Introduction}

Lately, the nanostructural process, which depends on the utilization of distinctive features of the nanostructural ranges from 1 to $\sim 100 \mathrm{~nm}$, the size of the different structural constituents such as layers, grains, pores and so on is utilized always for production of new materials and determine the physicochemical, non-equilibrium nature and the physic-mechanical characteristics of nanostructural materials. It is obvious from general searches that radiation, thermal and deformation impact, bringing about homogenization, recrystallization and other phenomena could be accompanied by the change in the nanostructure through the deterioration of its characteristics [1-2].

Chitosan is considered as a natural polymer (cationic polysaccharide) that extracted from chitin through the deacetylation process. Chitosan's ability to treat thin films or porous structures for use in diverse applications is one of the most characteristic features of chitosan [3-4]. Chitosan is used in pharmaceutical and medical applications such as surgical Sutures, sponges, artificial skin, drugs release, membranes and contact lenses and etc ... The characteristic of chitosan can be modified through the addition of inorganic material, organic substance, nanomaterial or another polymer [4-6]. The characteristics of chitosan can be adjusted through UV irradiation, heating and mechanical remediation. There are three steps occur during ultraviolet irradiation of polymer matrices. In the first step, the excited chitosan molecules are created, in the second step, a scission of the chain and the cross-link occurs then finally the oxidation state take place [7].

Many semiconductor nanomaterials such as $\mathrm{ZnO}, \mathrm{Fe}_{2} \mathrm{O}_{3}, \mathrm{TiO}_{2}, \mathrm{WO}_{3}, \mathrm{CuO}$, and $\mathrm{SnO}$ have considerable special attention in various applications [8]. Unfortunately, most of them have various disadvantages, which limit their use in practical applications such as heterogeneity on its structures, have a large particle size, inactive below the visible light and contain a high value in its band gap (for example $\mathrm{TiO}_{2}$ is nearly 3.2 $\mathrm{eV}$ ), as a result of the conventional methods of

*Corresponding author E-mail: a.m_abdelghany@yahoo.com

DOI: 10.21608/JTCPS.2018.5521.1007

C2018 National Information and Documentation Centre (NIDOC) 
preparation. Thus, the evolution and synthesis of new material are to reinforce the activation and utilization below visible light (solar light) [9-10].

Among those photocatalytic materials, silver vanadates are significant materials because they have an efficient photophysical propertied under solar light irradiation, electrochemical, chemical sensing properties, controlled nanocrystallinity, morphology and a narrow band gap. Previously, AgVO3 nanomaterials have been synthesized by different techniques including co-precipitation, biomimetic method, sonochemical synthesis and hydrothermal process. Silver vanadate $(\beta-\mathrm{AgVO} 3)$ nanorods are used in various applications such as antibacterial agents, in lithium-ion batteries as cathode substance, photocatalysts and in the electrochemical cells [1113]. Recently, hybrid components based on chitosan have been progressed, including metal nanoparticles, conducting polymers and metal oxide nanoparticles because of their excellent characteristics of individual materials and simultaneous biocompatibility effects. Hence, the incorporation of metal oxide nanomaterials into chitosan to produce materials of outstanding photocatalytic effect and stable in both acidic and basic solvents attracted much attention of many researchers [14-16]. Organic and inorganic blockers are used to protect from UV radiation, but organic blockers absorb UV rays. The inorganic blockers such as $\mathrm{TiO} 2, \mathrm{AgVO} 3$ and $\mathrm{ZnO}$ are common choices to scatter UVA and UVB radiation. Then, inorganic blockers can be used as a sun protection cream to prevent the skin cancer [17].

Chitosan and silver vanadate nanorods can form the synthetic composites due to their specific characteristic of both components. The prepared composites confer specific chemical, mechanical and structural characteristics. The production of new biomaterials with relatively low pollution, unique properties and low cost has a great possibility to be used in various applications [15].

The main goal of present study devoted for preparation and characterization of chitosan filled AgVO3 nanorods using a simple casting method and determines the effect of variable filler concentrations on the optical and surface characteristics of prepared samples for different optical and industrial applications.

\section{Experimental}

\section{Materials and Methods}

Chitosan was obtained from (Aldrich Co.). Silver vanadate was obtained from the reaction of
$0.01 \mathrm{M}$ ammonium trioxovanadate (NH4VO3) that purchased from Sigma-Aldrich, Germany and 0.01 $\mathrm{M}$ silver nitrate $(\mathrm{AgNO} 3)$ that obtained from Bio Basic Canada Inc.

The accurate amount of AgNO3 (0.01 M) was dissolved in $200 \mathrm{ml}$ distilled water and added drop by drop to NH4VO3 $(0.01 \mathrm{M})$ that dissolved in $200 \mathrm{ml}$ distilled water. The mixture of the solution was stirred at ambient temperature and controlled $\mathrm{pH}$ from 4.6 to 5.8. AgVO3 was observed by the production of a yellow precipitate. Silver vanadate nanorods were washed by using ethanol and distilled water several times and kept thermally in the oven at $50 \circ \mathrm{C}$.

Polymeric composites were prepared through the mixing of different concentrations of silver vanadate with $1 \mathrm{~g}$ chitosan that dissolved in $2 \%$ acetic acid. The latest composition of the prepared films was $(0.01,0.02,0.03,0.04,0.05,0.06 \mathrm{Wt}$. \%) of silver vanadate in the polymer composite.

The prepared films were obtained by casting the solutions into plastic Petri-dishes .Polymer films were submitted to the ultra-high sonication instrument at room temperature for $15 \mathrm{~min}$.After solvent evaporation, the prepared thin films were kept thermally in the oven at $50{ }^{\circ} \mathrm{C}$ and placed in a vacuum desiccator at room temperature until use. Table (1) summarizes the different concentration of the prepared films.

\section{Characterization}

The spectrophotometer (V-570 UV/VisNIR, JASCO, Japan) was employed to measure Ultraviolet/visible (UV/Vis) absorption spectra in the wavelength region of 190-1100 nm.

\section{$\underline{\text { Results and Discussion }}$}

\section{Ultra violet -visible radiation}

$\mathrm{UV}$-Vis spectroscopy is an important analytical method that consists of an absorption range

TABLE 1. The composition of chitosan filled with $\mathrm{AgVO}_{3}$ polymer films.

\begin{tabular}{ccc}
\hline Samples & Chitosan (wt. \%) & AgVO $_{\mathbf{3}}(\mathbf{w t . \%})$ \\
\hline Cs & 100.0 & 0.00 \\
Cs1 & 99.99 & 0.01 \\
Cs2 & 99.98 & 0.02 \\
Cs3 & 99.97 & 0.03 \\
Cs4 & 99.96 & 0.04 \\
Cs5 & 99.95 & 0.05 \\
Cs6 & 99.04 & 0.06 \\
\hline
\end{tabular}


from the $190-1100 \mathrm{~nm}$ to identify the functional groups in molecules due to excitation of electrons according to molecular orbital theory.

The UV-visible spectroscopy of the prepared $\mathrm{AgVO} 3$ nanorods is shown in Fig. (1). The steep curve of the spectra analysis observes that the visible light absorption that because of the bandgap transition. The absorption band of prepared silver vanadate nanorods at room temperature is equal $376 \mathrm{~nm}$. In $\mathrm{AgVO}$, the valence band has consisted of Ag $4 \mathrm{~d}$ and $\mathrm{O} 2 \mathrm{p}$ orbitals. The properties of absorption edge indicated for $\mathrm{AgVO} 3$ is due to the hybrid orbital of $\mathrm{Ag} 4 \mathrm{~d}$ and $\mathrm{O} 2 \mathrm{p}$ or (the transition of electrons from the $\mathrm{Ag}$ $4 \mathrm{~d}$ and $\mathrm{O} 2 \mathrm{p}$ ) in valence band orbitals to vacant $3 \mathrm{~d}$ conduction band of $\mathrm{V} 5+$, which observe a high donating of $\mathrm{V} 3 \mathrm{~d}$ and $\mathrm{Ag} 5 \mathrm{~s}$ orbitals that located in the lower section of the conduction band.

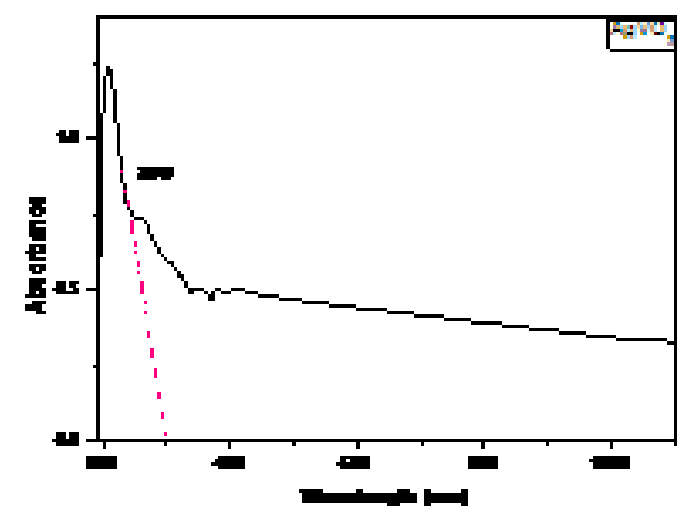

Fig. 1. UV/Vis analysis of synthesized AgVO3.

The optical energy band gap (Eg value) can be determined from plot photon energy (hv) versus $(\alpha \mathrm{h} v) 2$. The optical energy band gap (Eg) can be estimated through the intercept of the tangent in the $\mathrm{X}$-axis that shown in Fig.(2). Eg value is equal to $2 \mathrm{eV}$ for $\mathrm{AgVO}$. After the semiconductor is excited, the recombination processes are associated with distortions in the crystalline lattice. Therefore, the electronic characteristic can be altered due to the structural distortions. In our case, the emissions are very sentient to alter in the primary coordination sphere of $\mathrm{V}$ and $\mathrm{Ag}$ cations and observe different changes in the excitation spectra compared to their normal structural.

The photocatalytic activity depends on transfer, creation and segregation of photogenerated electron-hole pairs. The position of the band was calculated by the next empirical formulas:



Fig. 2. Plots of (ahv)2 versus photon energy (hv) for the band gap energy of $\mathrm{AgVO3}$.

$$
\begin{aligned}
& E_{\mathrm{VB}}=\mathrm{X}-\mathrm{E}^{\mathrm{e}}+0.5 \mathrm{E}_{\mathrm{g}} \\
& \mathrm{E}_{\mathrm{CB}}=\mathrm{E}_{\mathrm{VB}}-\mathrm{E}_{\mathrm{g}}
\end{aligned}
$$

Where $E V B$ is the edge potential of valence band, $X$ is the electronegativity of transition metal oxide semiconductor that the geometric signify to the electronegativity of atoms, $E e$ is the energy of free electrons on the hydrogen scale (approximately $4.5 \mathrm{eV}$ ), ECB is the edge potential of conduction band and $E g$ is the energy of band gap for transition metal oxide semiconductor. The $X$ value of AgVO3 is $5.86 \mathrm{eV}$. As indicated in Table (2), according to the calculation, the values of $E g$ of the valence band and the conduction band of silver vanadate nanorods are $2.35 \mathrm{eV}$ and $0.53 \mathrm{eV}$, respectively, which is comparable to the values described in the literature [18].

Figure (3) shows the absorption analysis of pure chitosan and prepared thin films. It can be observed that the pure chitosan presence in the invisible region at $226 \mathrm{~nm}$ due to its transparency. The absorption spectra indicated the transition of existed electrons form $n \rightarrow p^{*} d$ orbital due to the presence of auxochromes functional groups (NHCOR) and $\pi \rightarrow \mathrm{p}^{*}$ transition due to the presence of chromophores groups $(\mathrm{C}=\mathrm{O})$ or $(\mathrm{C}=\mathrm{C})$ with a minimum excitation energy.

Whereas the prepared thin films that filled with a different concentration of silver vanadate nanorods indicate the shift of peaks to the longer wavelength in the range from $226-446 \mathrm{~nm}$, Based on UV-visible analysis, the raising of the relative intensity is due to the incorporation of silver vanadate nanorods into pure chitosan matrix and the strong complexation and the interaction between $\mathrm{AgVO} 3$ and polar group of pure chitosan matrix. In the prepared system, the formation of $\mathrm{AgVO} 3$ nanorods can be observed from the change of color of the polymer composites from colorless to yellow. 
TABLE 2. Summarizes the different the values of $E_{g}$ of the valence band and the conduction band of silver vanadate nanorods.

\begin{tabular}{ccccc}
\hline \multirow{2}{*}{ Sample } & \multicolumn{4}{c}{ Optical property $(\mathrm{eV})$} \\
\cline { 2 - 4 } & $\boldsymbol{E}_{g}$ & $\boldsymbol{E}_{V B}$ & $\boldsymbol{E}_{C B}$ \\
\hline n-AgVO $_{3}$ & 2 & 2.35 & 0.35 \\
\hline
\end{tabular}

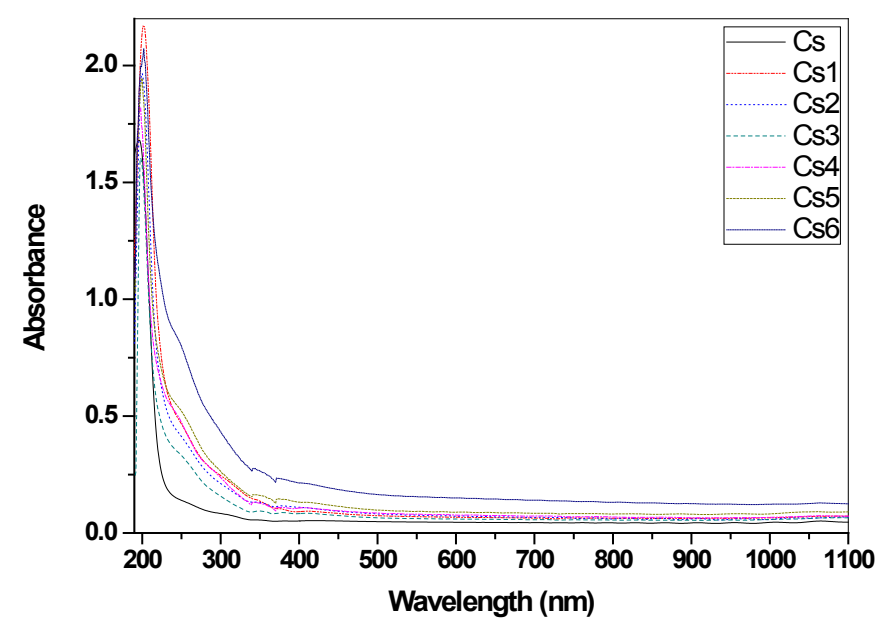

Fig. 3. UV-Visible absorption spectra for pure chitosan and prepared thin films.

From the equation of Einstein's photon energy, the energy of the conduction band (Ecb) can be estimated directly:

$$
E c b=h c / \lambda \max
$$

Where $\lambda \max$ is the maximum of UV-visible absorbance wavelength, $\mathrm{c}$ is the speed of light and $\mathrm{h}$ is the Planck constant. The results are tabulated in Table (3). From the UV-visible analysis, it can be observed a wide peak in some of the absorption spectra. Thus, the energy of the conduction band of all prepared samples can be estimated indirectly by the Tauc's equation:

$$
\begin{aligned}
& (\alpha h v)^{2}=C\left(h v-E_{c b d}\right) \\
& (\alpha h v)^{\frac{1}{2}}=C\left(h v-E_{c b i}\right)
\end{aligned}
$$

Where Ecb is the direct energy of the conduction band (Ecb), $\mathrm{C}$ is constants, $\mathrm{h}$ is Planck's constant, hu is the photon energy and $\alpha$ is the absorption coefficient. The direct and indirect transition energy of the conduction band of all prepared samples can be shown in Figure (4) and Figure (5) respectively. It is evident from the Figure (4), by increasing the concentration of silver vanadate nanorods, the energy of the conduction band (Ecb) decreased from 5.82 to $4.16 \mathrm{eV}$ for direct transition and from 5.28 to 1.84 for indirect transition respectively. This denotes that silver vanadate nanorods formed a localized state and the density of this state increased by increasing the concentration of $\mathrm{AgVO} 3$ nanorods, thus the energy of band gap decreased.

Urbach energy can determine the degree of material imperfection such as amorphous materials with a low crystalline in nature as shown in Fig. (6) and Fig.(7). Urbach energy can be calculated through the following equation: atiEquon (6) is missing (6)

Where is the absorption coefficient, is a constant, ho is the photon energy and $\mathrm{Eu}$ is the Urbach energy that listed in the Table (3).

\section{Refractive index}

Changes in the value in the refractive index of all prepared thin films are important for controlling the optical characteristics of materials. Refractive index is a crucial optical parameter for designing optical fibers, prisms and optical windows. The refractive index value of the polymer composites are calculated using [19]:

$$
(\mathrm{n} 2-1) /(\mathrm{n} 2+2)=1-
$$

Where $\mathrm{n}$ is the value of the refractive index and is the indirect energy of the conduction band 
TABLE 3. the optical properties of all prepared thin films.

\begin{tabular}{cccccc}
\hline \multirow{2}{*}{ Samples } & $\begin{array}{c}\text { edge } \\
(\mathbf{n m})\end{array}$ & $\begin{array}{c}\mathbf{E}_{\mathbf{c b}}(\mathbf{e V}) \\
\text { by equation (3) }\end{array}$ & $\begin{array}{c}\mathbf{E}_{\text {cbd }}(\mathbf{e V}) \\
\text { by equation (4) }\end{array}$ & $\begin{array}{c}\mathbf{E}_{\text {cbi }}(\mathbf{e V}) \\
\text { by equation (5) }\end{array}$ & $\begin{array}{c}\mathbf{U}_{\text {energy }} \\
\end{array}$ \\
\hline Cs & 226 & 5.48 & 5.82 & 0.50 \\
Cs1 & 259 & 4.78 & 5.68 & 5.28 & 0.60 \\
Cs2 & 278 & 4.44 & 5.76 & 5.08 & 0.60 \\
Cs3 & 289 & 4.28 & 5.85 & 5.23 & 0.80 \\
Cs4 & 319 & 3.87 & 5.90 & 5.20 & 0.79 \\
Cs5 & 322 & 3.85 & 5.86 & 5.20 & 0.85 \\
Cs6 & 342 & 3.61 & 5.70 & 4.88 & 1.01 \\
\hline
\end{tabular}



Fig. 4. The relation between $(h) 2$ versus photon energy $(h)$.

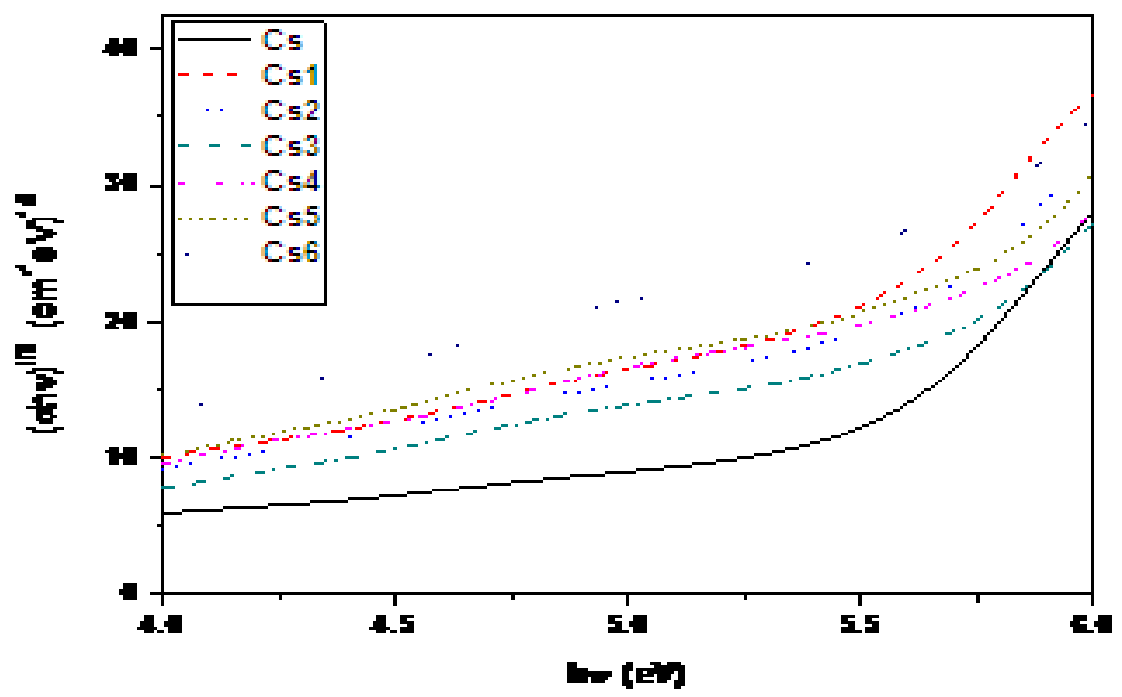

Fig. 5. The relation between (h)1/2 versus photon energy (h). 




Fig. 6. Uenergy for pure chitosan.

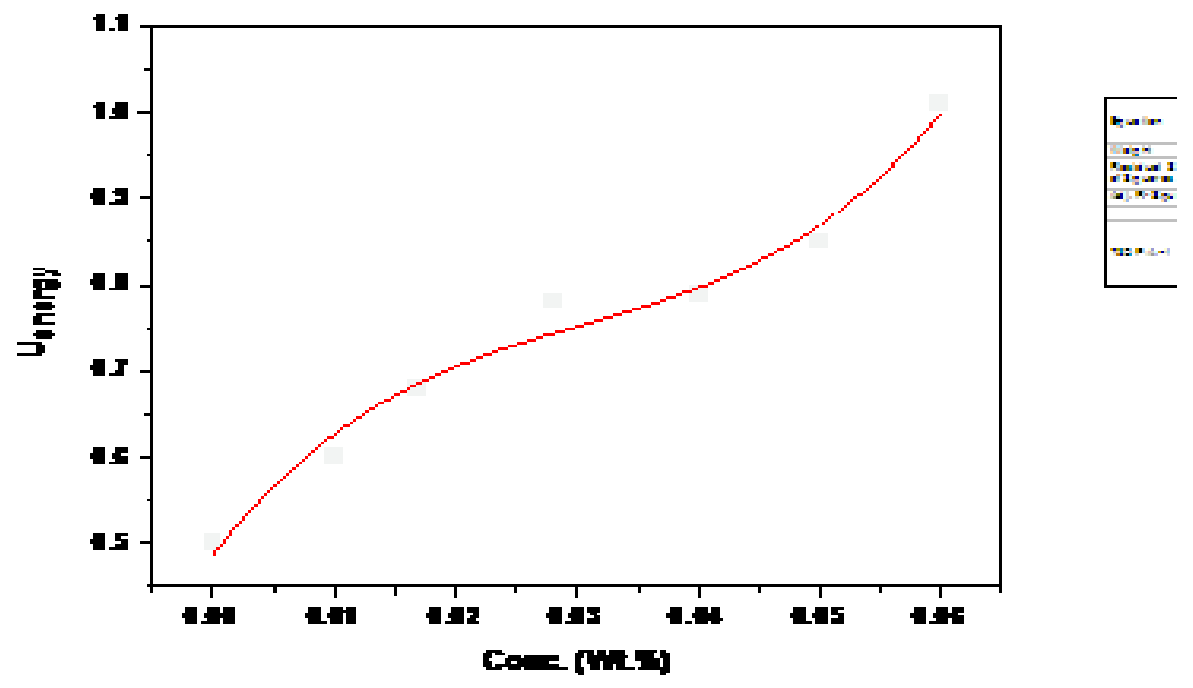

Fig. 7. The relation between Uenergy and different concentration of polymer nanocomposites.

TABLE 4. refractive index for all prepared samples.

\begin{tabular}{cc}
\hline Samples & Refractive index (n) \\
\hline Cs & 1.95 \\
Cs1 & 1.97 \\
Cs2 & 1.98 \\
Cs3 & 1.94 \\
Cs4 & 1.97 \\
Cs5 & 1.97 \\
Cs6 & 2.00 \\
\hline
\end{tabular}

J.Text.Color. Polym. Sci., Vol. 15, No.1 (2018) 
gap. The refractive index value of the polymer composites is listed in Table (4). It's clear that the value refractive index raised from 1.95 for pure chitosan to 2.77 for chitosan incorporated of the maximum concentration of $\mathrm{AgVO} 3$ nanorods. The chitosan matrix becomes denser due to the incorporation of $\mathrm{AgVO} 3$ nanorods and therefore display a high refractive index. Polymer composites with the high value of refractive index can be used in several applications involving light-emitting diodes, waveguides and antireflective coatings in optoelectronic and the optical instruments. It is obvious that the value of the refractive index is raised by increasing the concentration of $\mathrm{AgVO} 3$ nanorods and indicated the homogeneous distribution of $\mathrm{AgVO} 3$ nanorods within the chitosan matrix.

\section{Conclusion}

Silver vanadate nanorods have been successfully prepared via co-precipitation method by using deionized water, ammonium trioxovanadate and silver nitrate as the ingredients. Incorporation of $\mathrm{AgVO} 3$ nanorods into pure chitosan film through solution casting method produced the chitosan - $\mathrm{AgVO} 3$ thin film. The immobilized AgVO3 showed the strong incorporation and the homogenous distribution within chitosan film. The UV absorption analysis is shown for polymer nanocomposites. The wavelength increases by the addition of $\mathrm{AgVO} 3$ nanorods. Optical band gap decreases by the addition of silver vanadate nanorods loading. The enhancement in conducting properties is observed for all prepared thin films, which is illustrated from Tauc's plot.

\section{References}

1. Shah, S. I.; Powers, T. M. Nanotechnology A path forward for developing nations. IOP Science. 92, 54-65 (2015).

2. Mackerle, J. Nanomaterials, nanomechanics and finite elements: a bibliography. IOP Science. 13, 1-162 (2004).

3. Branca, C.; D`Angelo, G.; Crupi, C.; Khouzami, K.; Rifici, S.; Ruello; G.; Wanderlingh. U. Role of the $\mathrm{OH}$ and $\mathrm{NH}$ Vibrational groups in polysaccharidenanocompsite interactions: A FTIR-ATR study on Chitosan and chitosan lclay films. Polymer. 99, 614-622 (2016).

4. Azuma, K.; Izumi, R.; Osaki, T.; Ifuku, S.;
Morimoto, M.; Saimoto, H. Chitin, chitosan, and its derivatives for wound healing: old and new materials. Journal of functional biomaterials. 6, 42-104 (2015).

5. Lan, G.; Lan, G.; Wang, T.; Wang, L.; Chen, J.; Yu, K.; Liu, J.; Dai, F.; Wu, D. Chitosan/ gelatin composite sponge is an absorbable surgical hemostatic agent. Colloids and Surfaces B: Biointerfaces. 136, 1026-1034 (2015).

6. Pillai, C. K. S.; Paul, W.; Sharma, C. P. Chitin and chitosan polymers: Chemistry, solubility and fiber formation. Progress in Polymer Science. 34, 641678 (2009)

7. Sionkowska, A.; Wisniewski, M.; Skopinska, J.; Vicini, S.; Marsano, E. The influence of UV irradiation on the mechanical properties of chitosan/poly (vinyl pyrrolidone) blends. Polymer Degradation and Stability. 88, 261-267 (2005).

8. Zavala, F. G.; Alarcón, L. E.; Casados, D. A. S.; Pesqueira, M. E.; Poniatowski, E. H.; Castellón, E. R.; Aguado, E. R. Synthesis and characterization of silver vanadates thin films for photocatalytic applications. Catalysis Today. 305, 102-107 (2018).

9. Holtz, R. D.; Lima, B. A.; Filho, A. G. S.; Brocchi, M.; Alves, O. L. Nanostructured silver vanadate as a promising antibacterial additive to water-based paints. Nanomedicine: Nanotechnology, Biology and Medicine. 8 935-940 (2012).

10. Hu, X.; Hu, C.; Qu, J. Preparation and visiblelight activity of silver vanadate for the degradation of pollutants. Materials Research Bulletin. 43, 2986-2997 (2008).

11. Sharma, S.; Panth öfer, M.; Jansen, M.; Ramanan, A. Ion exchange synthesis of silver vanadates from organically templated layered vanadates. Materials Chemistry and Physics. 91, 257-260 (2005).

12. De Castro, D. T.; Valente, M. L. C.; De Silva, C. H. L.; Watanabe, E.; Siqueira, R. L.; Schiavon, M. A.; Alves, O. L.; dos Reis, A. C. Evaluation of antibiofilm and mechanical properties of new nanocomposites based on acrylic resins and silver vanadate nanoparticles. Archives of Oral Biology. 67 46-53 (2013).

13. Mohandes, F.; Niasari, M. S. Sonochemical synthesis of silver vanadium oxide micro/ nanorods: Solvent and surfactant effects. Ultrasonics Sonochemistry. 20, 354-365

J.Text.Color. Polym. Sci., Vol. 15, No.1 (2018) 
(2013).

14. Hamden, Z.; Bouattour, S.; Ferraria, A. M. ; Ferreira, D. P. ; Ferreira, L. F.V. ; Rego, A. M. B.; Boufi, S. In situ generation of $\mathrm{TiO} 2$ nanoparticles using chitosan as a template and their photocatalytic activity. Journal of Photochemistry and Photobiology A: Chemistry. 321, 211- 222 (2016).

15. Vijayalekshmi, V. UV- Visible, Mechanical and Anti-Microbial Studies of Chitosan Montmorillonite Clay / TiO2 Nanocomposites. Research Journal of Recent Sciences. 4, 131-135 (2015).

16. Norranattrakul, P.; Siralertmukul, K.; Nuisin, R. Fabrication of chitosan/titanium dioxide composites film for the photocatalytic degradation of dye. Journal of Metals, Materials and Minerals. 23, 9-22 (2013).

17. Mitchnick, M. A.; Fairhurst D.; Pinnell, S. R. Microfine zinc oxide (Z-Cote) as a photostable UVA/UVB sunblock agent. Journal of the American Academy of Dermatology. 40, 85-90 (1999).

18. Lin, X.; Guo, X.; Shi, W.; Guo, F.; Zhai, H.; Yan, Y.; Wang, Q. Ag3PO4 quantum dots sensitized AgVO3 nanowires: A novel Ag3PO4/AgVO3 nanojunction with enhanced visible-light photocatalytic activity. Catalysis Communications. 66, 67-72 (2017).

19. Aziz, S. B.; Morphological and Optical Characteristics of Chitosan $(1-\mathrm{x})$ :Cuox $(4 \leq$ $\mathrm{x} \leq 12)$ Based Polymer Nano-Composites: Optical Dielectric Loss as an Alternative Method for Tauc's Model. Nanomaterials. 12, 444 (2017).

(Rreceived 8/10/2018, accepted 21/10/2018 ) 


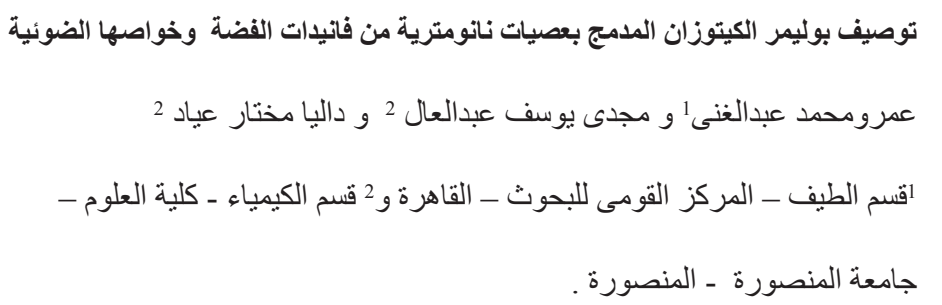

الكيتوزان عبارة عن بوليمر طبيعي متعدد السكريات وغير سام وقابل للتحلل كاتيوني ومتو افق حيويًا. العمل الحالي يقدام

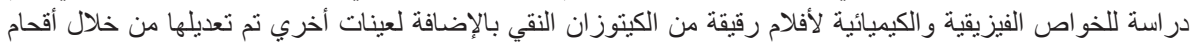

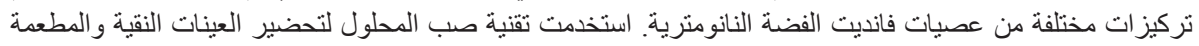

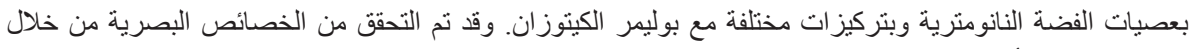

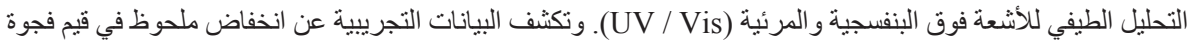

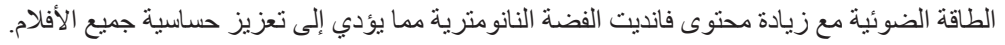

